\title{
A study of polypharmacy leading to adverse drug reactions in geriatric patients at tertiary care hospital
}

\author{
K. Chandra Mouli Krishna ${ }^{1}$, Swathi Ratnam Regidi ${ }^{2 \text {,* }}$ \\ ${ }^{1,2}$ Assistant Professor, Dept. of Pharmacology, ${ }^{1}$ NRI Institute of Medical Sciences, Visakhapatnam, Andhra Pradesh, ${ }^{2}$ Rajiv \\ Gandhi Institute of Medical Sciences, Srikakulam, Andhra Pradesh, India
}

*Corresponding Author:

Email: sweetyswathi12@gmail.com

\begin{abstract}
Polypharmacy is a common occurrence in elderly patients due to reasons like multiple co-morbdiities, multiple drug prescribing physicians. Adverse drug reactions (ADR) are most frequently observed among the elderly as the responsiveness to the drugs is altered due to number of age related confinement. In the present study we plan to identify the ADRs in elderly due to polypharmacy.

Objectives: 1. To assess the rationality of prescription based on WHO criteria and Beers criteria. 2. To identify the adverse drug reactions occurring in the elderly as a result of polypharmacy.

Materials and Methods: This prospective study was conducted in the department of General Medicine, Anil Neerukonda Hospital, attached to NRI Institute of Medical Sciences, Sangivalasa, Visakhapatnam. The study period was (Sep \& Oct) for 2 months in 2016. The patient prescriptions analyzed were 30. The inclusion criteria were $>65 y$ rs of both sexes and prescribed more than 5 drugs. The details of drugs collected were analyzed according to WHO/INRUD indicators.

Results: Study showed that, polypharmacy involving hypertension associated with diabetes and respiratory disorders receiving 10.4 drugs per patient. About 53.3\% of the patients received more than ten drugs per day. The most common medical diagnosis associated with polypharmacy was respiratory disorders with $39.7 \%$ and number of drugs was 10 in that group. About $18.9 \%$ of the drugs prescribed were potentially harmful drugs according to Beers list. The incidences of ADRs were $16.4 \%$ among the total patients, majority being type 1 ADRs.

Conclusion: The polypharmacy seen in majority of elderly patients was inappropriate. The average number of drugs per prescription was high $(>10)$. Many adverse drug reactions were noted according to beers list. The prescription pattern for geriatric age group should be by generic name and rational prescription should be followed.
\end{abstract}

Keywords: Adverse drug reaction, Geriatric, Polypharmacy.

\section{Introduction}

Polypharmacy is defined as concurrent use of multiple medications, greater than five by a single patient ${ }^{1}$. Responsiveness to drugs is modified among the elderly due to age-related changes such as decline in renal and liver function, hypoalbuminemia, reduced body weight, and multi morbidity which may increase the risk of polypharmacy leading to high frequency of adverse drug reactions among the elderly. ${ }^{1}$ Many studies have shown that inappropriate medication use in elderly as per Beer's list leads to increased morbidity and Mortality. ${ }^{2}$ Prevention of adverse drug reactions (ADR) and emphasis on patient safety belong to some of the top priorities for the Joint Commission for the Accreditation of Health Care Organizations. ${ }^{3}$ There are multiple factors associated with geriatric patient polypharmacy which have to be assessed, evaluated and have to be curtailed.

Factors associated with the development of polypharmacy in the geriatric population. ${ }^{3}$

1. Availability of nonprescription drugs

2. High cost of prescription medications (leads to use of up to several other "alternatives" to reach same effect)

3. Hoarding of old medications
4. Inadequate patient knowledge of medications and medical conditions

5. Increasing number of chronic health conditions

6. Patients being treated by multiple prescribing physicians

7. Patients using different sources of medication, often with little or no co-ordination between these sources

8. Taking at least one medication for every diagnosis

9. Tendency toward self-treatment/self-medication

\section{Aims and Objectives}

1. To assess the rationality of prescription based on Beers criteria $^{2}$ and WHO criteria ${ }^{4}$.

2. To identify the adverse drug reactions occurring in the elderly as a result of polypharmacy.

\section{Materials and Methods}

Study design: A prospective study.

Source of data:Department of General Medicine, Anil Neerukonda Hospital, attached to NRI Institute of Medical Sciences.

Study period: 2 months from September to October in 2016.

Sample Size: 30 patients were included in the study. 
Source of the Data: Patients records, medication history and where appropriate, discussion with the patient, patients attendants and physicians. Informed consent was taken from the selected patients before starting the study.

Inclusion criteria:

1. Patients $\geq 65$ years.

2. Patients who are on more than 5 drugs.

3. Patients who voluntarily gave informed consent.

Exclusion criteria:

1. Patients less than 65years

2. Elderly patients who are on $\leq 5$ drugs

3. Patients who did not gave voluntary informed consent.

Drug details:The drug details which includes name of the drug, dosage, duration of therapy, route of drug administration, reasons for polypharmacy, previous medication history were recorded.

\section{Statistical analysis}

At the end of the study, all data is compiled and statistically analysed using SPSS software version 21.The results were depicted in the form of percentages with tables and graphs.

\section{Results}

Study showed that, polypharmacy involving hypertension associated with diabetes and respiratory disorders receiving 10.4 drugs per patient. About 53.3\% of the patients received more than ten drugs per day. The most common medical diagnosis associated with polypharmacy was respiratory disorders with $39.7 \%$ and number of drugs was 10 in that group. About $18.9 \%$ of the drugs prescribed were potentially harmful drugs according to Beers list ${ }^{2}$. The incidence of ADRs was $16.4 \%$ among the total patients, majority being type 1 ADRs.

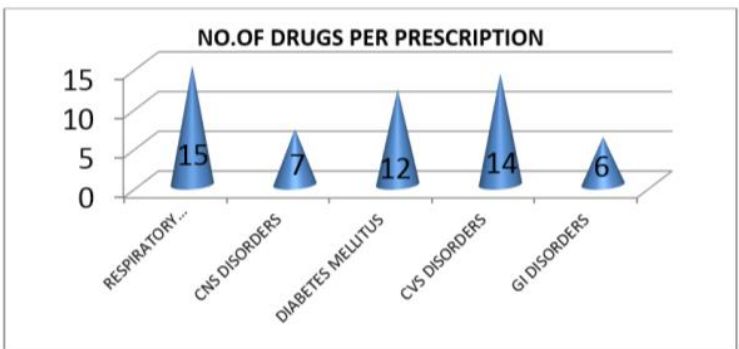

Fig. 1: Medical Diagnosis with Number of Drugs Per Prescription

The list of potentially harmful drugs prescribed for elderly as mentioned in Beer's list is given in Fig. 2. Patients were prescribed potentially harmful drugs according to Beer's list ${ }^{2}$. The drugs mainly prescribed in an in-appropriate manner according to Beer's listwere ferrous sulphate, salbutamol, aspirin, Chlorpheniramine Maleate, nifedipine.

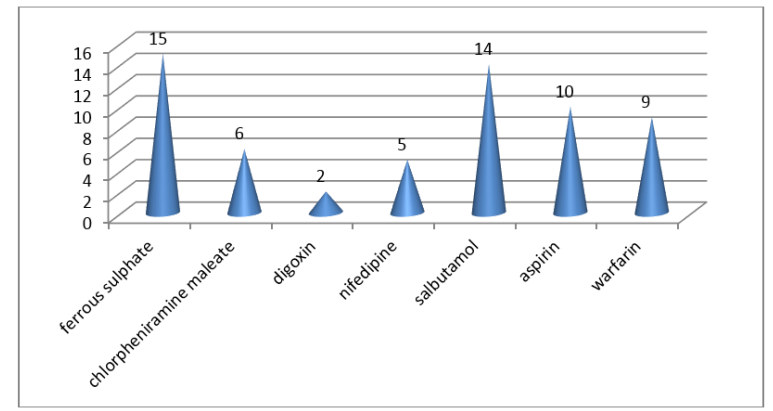

Fig.2 Potentially Harmful Drugs Prescribed As Per Beers List

Most commonly prescribed drugs in elderly are shown in Fig. 3. Most commonly prescribed drug was ranitidine in 26 patients, followed by diuretics like hydrochlorothiazide in 16 patients, followed by ferrous sulphate and paracetamol, each in 15 patients and finally salbutamol in 14 patients.

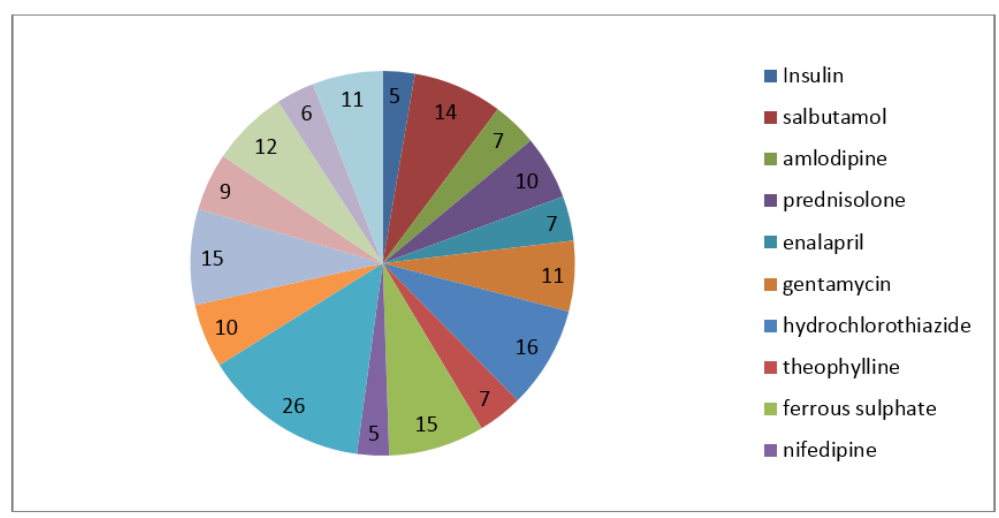

\section{Fig.3 Most Commonly Prescribed Drugs Which Cause Adverse Drug Reactions}

Irrational prescription of concurrent administration of the drugs noted in the study with theoretical reasons for irrationality is mentioned in Table No. 2. The drugs involved in majority of the drug interactions included ciprofloxacin, theophylline, ceftriaxone and azithromycin. 
Table 2: Irrational prescription of drug combinations and the reasons for irrationality

\begin{tabular}{|l|l|}
\hline Irrational drug combinations & Reasons for irrationality \\
\hline Gentamycin+furosemide & Increases ototoxicity \\
\hline Ceftriaxone+Acenocoumarol & Increases bleeding time \\
\hline Azithromycin+Theophylline & Increases plasma concentration oftheophylline \\
\hline Aspirin+Diclofenac & Gastritis \\
\hline Ramipril+Digoxin & $\begin{array}{l}\text { Ramipril increases the concentration } \\
\text { ofdigoxin }\end{array}$ \\
\hline Ceftriaxone+Azithromycin & Bactericidal + Bacteriostaticantagonism \\
\hline Enalapril+Aldactone & Hyperkalemia \\
\hline Ciprofloxacin+Theophylline & $\begin{array}{l}\text { Ciprofloxacin inhibits the metabolism } \\
\text { ofTheophylline }\end{array}$ \\
\hline
\end{tabular}

Adverse drug reactions noted in the study are mentioned in Table No. 3. Only certain and probable adverse drug reactions were taken for analysis. Adverse drug reactions were mainly seen for insulin in 5 patients, followed by salbutamol in 2 patients, digoxin in 2patients and amlodipine in 1 patient.

Table 3: Drugs which caused adverse drug reactions

\begin{tabular}{|l|c|c|}
\hline \multicolumn{1}{|c|}{$\begin{array}{c}\text { Adverse drug } \\
\text { reaction }\end{array}$} & Drug responsible & No. of patients \\
\hline Hypoglycaemia & Insulin & 5 \\
\hline Tremors & Salbutamol & 2 \\
\hline Palpitation & Digoxin & 2 \\
\hline Ankle edema & Amlodipine & 1 \\
\hline Dry mouth & Amitryptyline & 1 \\
\hline Cough & Enalapril & 1 \\
\hline Sedation & $\begin{array}{c}\text { Chlorpheniramine } \\
\text { Maleate }\end{array}$ & 1 \\
\hline Epistaxis & Acenocoumoral & 1 \\
\hline Tinitus & Gentamicin & 1 \\
\hline
\end{tabular}

\section{Discussion}

As polypharmacy increases the risk of ADRs \& inappropriate use of medication in elderly, efforts should be made to improve the prescribing practices. Our study results showed that out of 30 patients included in the study, $46.7 \%$ of them were males, $53.3 \%$ were females which are incontrary to a study shown in Indonesia where $66 \%$ of the patients were females. ${ }^{5}$ About $53.3 \%$ of the patients received more than ten drugs per day which is in contrary to a study conducted in Hong Kong where 68\% of the patients received less than four drugs. ${ }^{6}$ The study showed that respiratory disorders mainly exacerbation of COPD, pneumonia, upper respiratory tract infections were associated with polypharmacy followed by cardiovascular disorders mainly hypertension, CHF, coronary artery diseases, but cardiovascular disorders were found to be the main morbidity pattern observed by zaveri et al in india ${ }^{7}$. This difference could be attributed to the seasonal variation, as the incidence of respiratory disorders is high in the month of july \& Aug. The average number of drugs per prescriptions an important index of the standard of prescribing. The number of drugs per prescription in our study was 10.4 which is less compared to a similar study by Jochenschuler et $\mathrm{al}^{8}$ in Austria where the average number of drugs per prescription was 7.5.In our study, $16.4 \%$ of the patients were reported with ADRs. Prescribing by generic name can reduce the cost incurred on drugs and the risk of medication errors. Excess of prescribing by brand names may be considered as evidence of vigorous promotional activities by pharmaceutical companies. In our study, antibiotics were prescribed in $32.1 \%$ of the total drugs prescribed. But in a study conducted in elderly from India showed that $88.2 \%$ of the patients were prescribed antibiotics. $^{9}$ Therefore, rational prescription of antibiotics should be encouraged to prevent polypharmacy and antibiotic mis-use.

Most commonly prescribed drugs include Ranitidine followed by hydrochlorothiazide, paracetamol, and salbutamol. A study conducted in Austria ${ }^{8}$ showed that diuretics, Proton pumpinhibitors and ACE inhibitors were prescribed commonly. In this study, based on Beer's list ${ }^{2}$, $19.4 \%$ of total drugs were prescribed in an inappropriate manner which is higher than other two Indian studies which reported $7.42 \% \%^{9}$ and $4.1 \% .^{10}$ The Beer's list is a list of specific medications that are generally considered inappropriate when given to elderly people. For a wide variety of individual reasons, the medications listed tend to causeside effects in the elderly due to the physiologic 
changes of ageing. Inappropriately prescribed drugs like ferrous sulphate, chlorpheniramine maleate and salbutamol were seen in our study which is similar to other Indian studies also. ${ }^{7,11}$ Many studies have shown that ADRs are commonly seen in elderly. In our study, $16 \%$ of the patients were reported with ADRs. Most common ADRs were due to insulin, followed by salbutamol and digoxin. Another study by Harugeriet $\mathrm{al}^{11}$ showed that insulin, frusemide and prednisolone were responsible for majority of the ADRs in elderly. A study by Rupawala et $\mathrm{al}^{12}$ showed that antidiabetics, oral anticoagulants and antiplatelets lead to majority of ADRs in elderly. Many potentially hazardous concurrent use of drugs were also noted in the study as shown in table 3. Many interactions involved ciprofloxacin, azithromycin and theophylline. Majority of the drugs producing ADRs and these drugs are very commonly used in elderly. Greater care should be taken in prescribing them to decrease the associated morbidity and mortality in elderly patients.

\section{Conclusion}

This study has shown that Polypharmacy is common among the elderly. Polypharmacy was associated mainly with respiratory disorders in elderly. The polypharmacy seen in majority of elderly patients was inappropriate. The average number of drugs per prescription was high (which was more than 10). Many adverse events were noted according to beers list. The prescription pattern for geriatric age group should be by generic name and rational prescription should be followed. Healthcare professionals should be aware of the risks and fully evaluate all medications at each patient visit to prevent polypharmacy from occurring. Drug information services also should be set up in the hospitals to provide information about adverse effects and drug-drug interactions to the physicians.

\section{References}

1. Ayesha R, LaxminarayanaK.Polypharmacy leading to adverse drug reactions in elderly in a tertiary care hospital. Int J Pharm Bio Sci 2012;3(3):218-224.

2. American Geriatrics Society 2012 Beers Criteria Update Expert Panel. American Geriatrics Society updated Beers Criteria for potentially inappropriate medication use in older adults. J Am GeriatrSoc 2012;60:616-31.

3. Stawicki SP, Gerlach AT. Polypharmacy and medication errors: stop, listen, look and analyse, OPUS 12 scientist, 2009:3(1);6-10.

4. World Health Organization. Model list of essential medicines, 2010[cited 2018 April 20];16:131.Available from:http//www.who.int/medicines/publications/essentialme dicines/en/index.html

5. Rahmawati F, Pramantara IDP, RomahW, Sulaiman SAS. Polypharmacy and unnecessary drug therapy on geriatric hospitalised patients in Yogyakarta Hospital, Indonesia, International Journal of Pharmacy and Pharmaceutical Sciences, 2009:1(1);6-11.

6. Ko CF, Ko PS, Tsang ML.A survey on polypharmacy and use of inappropriate medication in a geriatric outpatient clinic, J HK Geriotr Sot,1996;7:28-31
7. Zaveri HG, Mansuri SM, Patel VJ. Use of potentially inappropriate medicines in elderly: A prospective study in medicine out patient department of a tertiary care teaching hospital, Indian journal of Pharmacology, 2010:42(2);95-98.

8. Schuler J, Duckelmann C, Beindl W, PrinzE, Michalski T, Pichler $\mathrm{M}$ et al., Polypharmacy and inappropriate prescribing in elderly internal-medicine patients in Austria, Wien KlinWochenschr, 2008:120;733-741.

9. Mandavi, Tiwari P. Profile of Pharmacotherapy in elderly Indian patients; Preliminary finding, The international Journal of Risk and safety Medicine, 2006:18;151-157.

10. Shenoy S. Evaluation of drug prescribing pattern in the elderly patients in tertiary care hospital, Indian journal of Pharmacology, 2006:38;96-98.

11. Harugeri A, Joseph J, Parthasarathi G, Ramesh M, Guido S. Potentially inappropriate medication use in elderly patients: A study of prevalence and predictors in two teaching hospitals. J Postgrad Med 2010;56:186-91.

12. Rupawala AH, Kshirsagar NA, Gogtay NJ.A retrospective analysis of adverse events in the elderly in a tertiary referral center in Mumbai(Bombay),India, Indian J Med Sci, 2009;63(5):167-173 . 